\title{
Myriad of Otorhinolaryngology Diseases Presenting as Unilateral Proptosis
}

\author{
Parijat Joshi ${ }^{1}$, Murundi Basavarajaiah Bharathi ${ }^{2}$, Ambale Rudrappa Babu $^{3}$
}

\begin{abstract}
Aim and objective: To describe the evaluation and management of multiple cases of unilateral proptosis due to various sinonasal pathologies. Materials and methods: Patients presenting to ENT OPD with unilateral proptosis were evaluated, diagnosed, and treated in this prospective study done over 2 years. All cases underwent complete otorhinolaryngological and ophthalmic examination apart from the endoscopic and radiological examination. Proptosis was measured by Hertel's exophthalmometry and readings above $21 \mathrm{~mm}$ or a difference of more than $3 \mathrm{~mm}$ between the two eyes were taken as positive. The cases were managed with appropriate surgery along with required medical management. Results: Twelve cases were observed over 2 years. The mean age of patients was $48.08 \pm 13.02$ years. Male:female ratio was 5:1. The most common cause was maxillary carcinoma with an orbital extension (four cases). Surgery was a mainstay of treatment in all patients, along with required medical treatment.

Conclusion: A good work up and with surgical management resulted in good recovery of the patients with only one mortality. Sinonasal infections accounted for $25 \%$ of cases, benign neoplasms and tumor-like conditions $33 \%$, and the rest $42 \%$ were constituted by malignant conditions.

Clinical significance: Assessment of proptosis in otorhinolaryngology is of paramount importance as it can be an early and/or only manifestation of nasal or paranasal sinus pathology. Although the incidence of proptosis in everyday ENT practice is exceedingly low, the knowledge of the important causes helps in clinching the correct diagnosis and timely management.

Keywords: Cavernous sinus thrombosis, Mucormycosis, Paranasal sinuses, Proptosis.

Clinical Rhinology An International Journal (2019): 10.5005/jp-journals-10013-1367
\end{abstract}

\section{INTRODUCTION}

The eye serves as a major crossroad for the central nervous system, nose, nasopharynx, paranasal sinuses, and all structures related to support and functioning of the globe. Proximal location of the orbit to nose and paranasal sinuses makes it vulnerable to affliction due to otolaryngological diseases, resulting in proptosis, one of the most common symptoms of orbital pathology. ${ }^{1}$ Proptosis is defined as a forward movement of the globe in relation to the skull and generally measured by a protrusion of the apex of the cornea in front of the outer orbital margin, while the eye looks straight ahead. Clinically significant proptosis is any forward displacement of the globe 3 $\mathrm{mm}$ greater when compared with the other eye. Normally, the measurement varies from 14 to $21 \mathrm{~mm}$, with the average value being slightly above $16 \mathrm{~mm}^{2}$ Measurements obtained over this denote some degree of proptosis. Proptosis poses to be an intriguing condition for the ENT surgeon as it can be caused as a result of various diseases located from the meninges to the paranasal sinuses and orbit. Proptosis even though an uncommon symptom in day-to-day practice needs a detailed evaluation and thorough management for a good outcome.

In this prospective study, we describe a study of multiple cases of unilateral proptosis cases due to various sinonasal pathologies along with their evaluation and management.

\section{Materials and Methods}

A total of 12 cases were observed, evaluated, and were managed both medically and surgically.

All patients with signs of unilateral proptosis due to lesions of nasal cavity and paranasal sinuses presenting to the outpatient block of the department of otorhinolaryngology and head and
${ }^{1-3}$ Department of ENT and Head and Neck, JSS Hospital, Mysuru, Karnataka, India

Corresponding Author: Parijat Joshi, Department of ENT and Head and Neck, JSS Hospital, Mysuru, Karnataka, India, Phone: +91 7406715004, e-mail: parijatjoshi.24@gmail.com

How to cite this article: Joshi P, Bharathi MB, Babu AR. Myriad of Otorhinolaryngology Diseases Presenting as Unilateral Proptosis. Clin Rhinol An Int J 2019;12(2 and 3):57-62.

Source of support: Nil

Conflict of interest: None

neck over 2 years were taken up for this prospective study while proptosis due to endocrine causes and primary orbital pathology were excluded. Tenets of the declaration of Helsinki (1964, amended 2013) were adhered to.

All the cases underwent complete otolaryngological work-up including clinical and endoscopic examination of the nose, nasopharynx, and paranasal sinuses and neck examination for neck masses.

All cases also underwent complete ophthalmological examination including visual acuity assessment, color vision, extraocular movements, and fundoscopy.

Proptosis was measured using Hertel's exophthalmometry and was defined as more than $21 \mathrm{~mm}$ or a difference of more than $3 \mathrm{~mm}$ between the two eyes as measured by the Hertel's exophthalmometry method.

All patients were investigated radiologically with computed tomography of the paranasal sinuses (CTPNS), magnetic resonance imaging (MRI) was performed to localize the lesion and evaluate 
the extent as an adjunct to surgery. The lesion was approached as per the anatomic location. Direct fine needle aspiration cytology (FNAC) from the mass was done whenever possible, ultrasonography (USG)-guided FNAC was done whenever required and preoperative biopsy was done whenever required. The cases of infectious etiology required appropriate surgery along with medical management. The malignancies and benign lesions were dealt with both radical and excisional surgeries as fitting for the lesion along with radiochemotherapy as adjuvants in case of malignancies. All patients were reviewed and followed up at 1 week, 3 weeks, 3 months, 6 months, and 1 year. Detailed otorhinolaryngology examination and evaluation of the proptosis was done on each follow-up visit.

\section{Results}

The mean age of patients was $48.08 \pm 13.02$ years (ranging from 22 to 70 years). Most patients were in the $41-50$ years age group $(n=6$, $50 \%)$. There were two patients (16.7\%) in the $21-30$ age group, one patient (8.3\%) in the $31-40$ age group, two patients (16.7\%) in the 51-60 age group, and one patient was in the above 60 years group.

Male:female distribution in our series was found to be 5:1 and the right eye was found to be involved in five cases (41.7\%) whereas the left eye was found to be involved in the rest of the seven cases (58.3\%).

Sinonasal infections accounted for $25 \%$ of our cases, benign neoplasms and tumor-like conditions formed $33 \%$, and the rest were constituted by malignant conditions (42\%). The exact etiologies are listed in Table 1.

The benign conditions included myxoma of the nasal septum ( $n=1)$, extracranial ethmoidal meningioma $(n=1)$, and frontal sinus mucocele $(n=1)$. Among the malignant conditions, the most common were the maxillary malignancies of which three cases were histopathologically proven to be adenoid cystic carcinoma, while one was squamous cell carcinoma (SCC). A rare entity, rhabdomyosarcoma of the nasal cavity extending to the orbit was also encountered which resulted in the only mortality of this study. Interestingly, this patient had an initial history of total maxillectomy for malignant inverted papilloma, operated 8 years ago for same. However, tissue diagnosis with immunohistochemistry done at this presentation proved the tumor to be rhabdomyosarcoma. One case of carotid cavernous sinus fistula was seen secondary to blunt trauma to the nose and left eye.

All patients had proptosis on initial presentation as their main complaint with proptosis varying from 3 to $6 \mathrm{~mm}$. An average proptosis of $4.25 \mathrm{~mm}$ was seen in our study with most patients having proptosis between 4 and $5 \mathrm{~mm}(n=6,50 \%)$. Four patients

Table 1: Etiological distribution of the patients

\begin{tabular}{ll}
\hline Diagnosis & No. of cases \\
\hline Carotid-cavernous sinus fistula & 1 \\
Rhinosinusitis complicated by cavernous sinus & 2 \\
thrombosis & 1 \\
Frontal sinus mucocele & 1 \\
Orbital apex syndrome & 3 \\
Adenoid cystic carcinoma of the maxilla & 1 \\
Squamous cell carcinoma of maxilla & 1 \\
Myxoma of nasal septum & 1 \\
Extracranial ethmoidal meningioma & 1 \\
Rhabdomyosarcoma &
\end{tabular}

had proptosis of $3 \mathrm{~mm}$ and two had proptosis of $5 \mathrm{~mm}$. The majority of the cases $(n=8,67 \%)$ had non-axial proptosis and were secondary to the mass effect.

Almost all patients had nasal symptoms along with proptosis, including nasal block and nasal discharge (10 patients each). The other symptoms included headache $(n=8)$, cheek swelling $(n=6)$, epistaxis $(n=5)$, and visible nasal mass $(n=4)$. The most common ophthalmological symptoms were restriction of extraocular movements in nine cases, while there was a decreased vision in seven cases, diplopia in five, and ptosis in four cases. The decreased visual acuity loss of the patients ranged from mild to total blindness.

Surgical management was the mainstay of treatment. Cases of rhinosinusitis complicated by cavernous sinus thrombosis $(n=$ 2 ) and orbital apex syndrome $(n=1)$ underwent endoscopic sinus surgery to restore the drainage of the infected sinuses along with broad-spectrum antibiotic coverage. In one case, the offending agent was found to be Mucor; hence, was treated with IV liposomal amphotericin B $5 \mathrm{mg} / \mathrm{kg} /$ day along with correction of underlying uncontrolled diabetes. Both nasal septum myxoma $(n=1)$ and extracranial ethmoidal meningioma $(n=1)$ underwent endoscopic resection after precise localization of the lesion with computed tomography (CT) and MRI scanning. All four cases of maxillary malignancy required total maxillectomy followed by radiotherapy, one patient requiring orbital reconstruction due to extensive orbital invasion. The frontal mucocele $(n=1)$ was drained with the external frontal sinusotomy procedure. Rhabdomyosarcoma case $(n=1)$ was treated with orbital exenteration with prosthesis placement followed by chemotherapy; however, the patient succumbed to the disease within 6 months of initial presentation. Endoscopic fistula closure $(n=1)$ was done for the carotid-cavernous sinus fistula case (Table 2).

Except for the one mortality, all cases showed improvement/ complete resolution of proptosis without any signs of recurrence of disease at 6 months of follow-up.

However, no improvement was seen in the visual acuity/color vision status.

\section{Discussion}

The eye is a major crossroad for the central nervous system, nose, nasopharynx, paranasal sinuses, results in the extension of disease from these locations into orbit and ultimately to proptosis. Various ENT diseases may manifest as proptosis and numerous such disorders have been described. The signs and symptoms of such lesions may be nonspecific and do not help directly in reaching a diagnosis. Also, many of the lesions cannot be examined directly since these are neither visible nor palpable; moreover, surgical exploration of the orbit is an invasive procedure, which may lead to various ophthalmic complications. ${ }^{1}$ The average volume of an

Table 2: Surgical management of the cases

\begin{tabular}{ll}
\hline Surgery & No. of patients \\
\hline Endoscopic resection & 2 \\
Endoscopic sinus surgery & 3 \\
Total maxillectomy & 3 \\
Total maxillectomy with orbital reconstruction & 1 \\
External frontal sinusotomy & 1 \\
Fistula closure & 1 \\
Orbital exenteration & 1 \\
\hline
\end{tabular}


adult orbit is $30 \mathrm{~mL}$. An increase of orbital volume by only $5 \mathrm{~mL}$ will

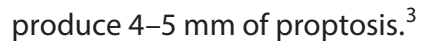

The incidence of tumors giving rise to unilateral proptosis varies in different age groups with some lesions occur in particular age groups, e.g., elderly patients are most commonly afflicted with malignancies. ${ }^{2}$ This study was done prospectively over 2 years and involves a description of 12 cases of unilateral proptosis presenting to the ENT OPD and underwent extensive diagnosis and management.

In our study, the mean age of the patients was $48.08 \pm 13.02$ years with most patients between 41 and 50 years of age group similar to the study by Venugopal and Sagesh ${ }^{4}$ who had studied 44 cases. Sinha et al. ${ }^{5}$ showed in a study of 50 consecutive cases of proptosis due to ENT causes showed a male:female ratio of 7:3, in comparison to our study where the majority of the cases were also males (M:F = 5:1).

The causes of proptosis are numerous. It may be due to neoplastic, vascular, traumatic, metabolic, or inflammatory in etiology. Orbit shares a common wall with a floor of the frontal sinus (roof of the orbit), lamina papyracea of an ethmoidal labyrinth (medial wall of the orbit), the root of the maxillary sinus (floor of the orbit), and anterolateral wall of the sphenoidal sinus (orbital apex). Any lesions in these regions have ready access to the orbital cavity. ${ }^{6}$

Most patients in our study had non-axial proptosis and were in the direction according to the origin. The direction of proptosis may indicate the site of the lesion. Tumors arising within the muscle cone-like optic nerve glioma may give purely axial exophthalmos. Diseases of frontal or ethmoid sinuses may displace the eyeball downwards and/or laterally. Medial displacement of the eye often indicates tumors of the lacrimal gland or temporal fossa. Tumors of the maxillary sinus may push the globe upward. ${ }^{7}$

In our study, all 12 (100\%) patients presented with complaints of one-sided proptosis while in a study of 20 patients by Rajenderkumar, ${ }^{2} 30 \%$ had initial complaints of proptosis and $20 \%$ did not complain of proptosis at all.

Wright ${ }^{8}$ in 1970 considered an absolute reading of more than 21 $\mathrm{mm}$ as proptosis. He observed that the average readings in males were that of $17 \mathrm{~mm}$, in females $16 \mathrm{~mm}$, and $14.5 \mathrm{~mm}$ in children. This study also took any reading above $21 \mathrm{~mm}$ or a difference of more than $3 \mathrm{~mm}$ between the two eyes as proptosis.

In our study, nasal discharge and nasal block were the most common ENT symptoms. The most common ophthalmological symptom after proptosis was found to be restriction of extraocular movements followed by visual loss while in the study by Sinha et al. ${ }^{5}$ Nasal block and epistaxis were commonest ENT complaints, though proptosis, reduced vision remained the most common ophthalmological symptoms. The nasal block was the most common symptom in the study by Venugopal and Sagesh ${ }^{4}$ as well. The nasal block is an important symptom and a thoroughly detailed examination should be done when patients present with the same. A study by Frazell and Lewis ${ }^{9}$ of 416 cases of malignancies of the nose and paranasal sinuses showed nasal block (35\%), facial swelling (25\%), and epistaxis (12.5\%) as the presenting complaints.

Calcaterra et al. ${ }^{7}$ stated that patients with unilateral proptosis warrant a systemic and multidisciplinary workup before surgical exploration or therapeutic regimen is undertaken. The otolaryngologist is concerned primarily with the diagnosis of the disease process that arises adjacent to the orbital space in the nose, paranasal sinuses, nasopharynx, and temporal fossae. Careful head and neck examination should be supplemented by recently refined investigations such as computed tomography, arteriography with subtraction, orbital venography, contrast orbitography, USG, etc. Computed tomography scan with its high positivity rate is of primary importance in the diagnostic workup and helps in determining any bony lesions but MRI is more helpful in detecting the exact morphology of the lesion and its extent.

In a case series of 17 cases of unilateral proptosis by Zaidi, ${ }^{10} 6$ out of the 17 cases were due to benign lesions. Of these six, four (23.5\%) were due to ethmoid polyposis, one (6\%) due to fibrous dysplasia, and one (6\%) due to Ringert's tumor. In contrast, we found $42 \%$ of lesions were malignant in our series $33 \%$ were due to benign neoplasms and tumor-like conditions. There was $41.7 \%$ right-sided and $58.3 \%$ left-sided proptosis which can be compared with the study of Rajenderkumar ${ }^{2}$ who found $50 \%$ right-sided proptosis and $45 \%$ left-sided and one case with bilateral proptosis. He also found a maximum of $8 \mathrm{~mm}$ of proptosis; however, in the present study, maximum of $6 \mathrm{~mm}$ was recorded.

A total of 79 cases of paranasal sinus tumors were reviewed by Johnson et al. ${ }^{11}$ Of these 47 (59\%) demonstrated orbital involvement out of which 18 (38\%) patients had proptosis and 16 (34\%) had nasal obstruction as a presenting complaint. The maxillary sinus was the most common site of origin of paranasal sinus tumors (61\%). Similar to his study, in our study out of seven paranasal sinus tumors, the commonest site of origin of the lesion was from the maxillary sinus (four cases), two from ethmoid sinuses, and in one origin could not be determined. In 10-year retrospective study of malignant tumors of the maxillary sinus, Popovic and Milisavljevic ${ }^{12}$ found that out of a total of 20 patients all were of SCC. Symptoms initially included nasal obstruction, epistaxis, etc. Three patients with orbital involvement presented with proptosis. Surgery with postoperative radiation remains the standard treatment for respective carcinoma.

Venugopal and Sagesh ${ }^{4}$ also found that SCC was the most common histological type constituting $58.4 \%$ of the cases in his case series of 44 cases followed by adenoid cystic carcinoma whereas, in our series, out of 4 maxillary carcinoma cases, 3 were histopathologically adenoid cystic carcinoma (Fig. 1).

Out of all 12 cases, 3 (25\%) were benign masses including extracranial ethmoidal meningioma, myxoma of the nasal septum, and frontal sinus mucocele, in a study by Rajenderkumar, ${ }^{2}$ out of 20 cases, $25 \%$ were mucocele, pyocele, $10 \%$ fibrous dysplasia, $5 \%$ frontal osteomas, and 5\% nasopharyngeal angiofibroma. All patients were managed surgically with endoscopic resection of nasal masses and external frontal sinusotomy (Fig. 2).

One of our patients presented with unilateral proptosis, frozen eyeball movements with uncontrolled diabetes. Magnetic resonance imaging brain showed ethmoid and sphenoid sinusitis with the extension of inflammatory changes into right cavernous sinus causing thrombosis with thrombosis of a cavernosal portion of right ICA. Mucormycosis was proven on histopathology of sphenoid sinus contents. This patient was treated with endoscopic sinus surgery to restore drainage of the sphenoid sinus along with intense medical management of uncontrolled diabetes mellitus with liposomal amphotericin B ( $5 \mathrm{mg} / \mathrm{kg} /$ day). In a study by Bhansali, ${ }^{13}$ mucormycosis was the presenting manifestation in one-fourth of the patients for underlying diabetes (Fig. 3).

Another intriguing presentation was of a patient with acute onset of proptosis, restricted extraocular movements, and loss of vision in the left eye. This was preceded by a history of left upper molar dental extraction 8 days ago for caries. It was followed by progressive swelling over the left cheek, trismus, and congestion 

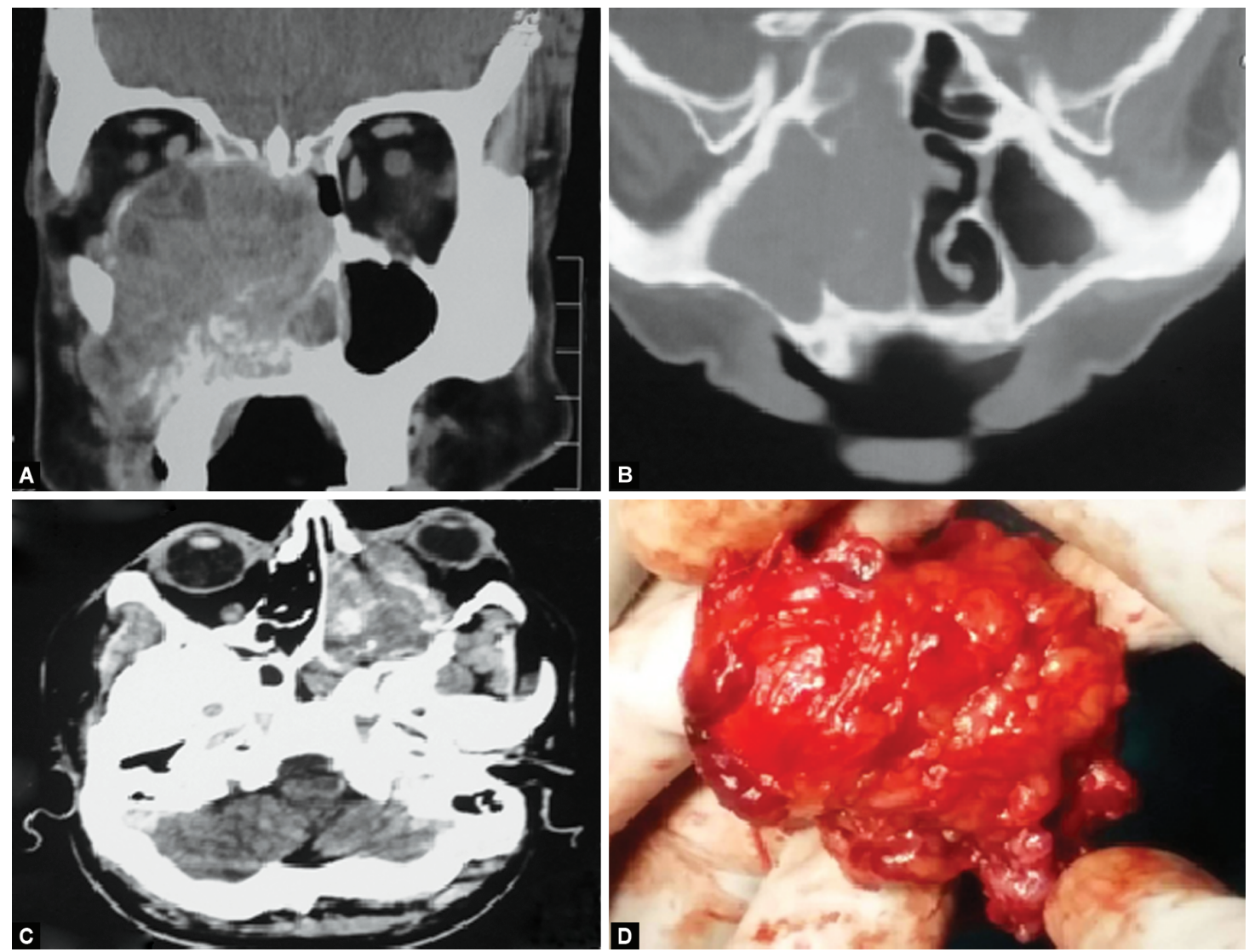

Figs 1A to D: (A to C) CT scan images of cases of adenoid cystic carcinoma maxilla; (D) Specimen of exenterated tumor of rhabdomyosarcoma
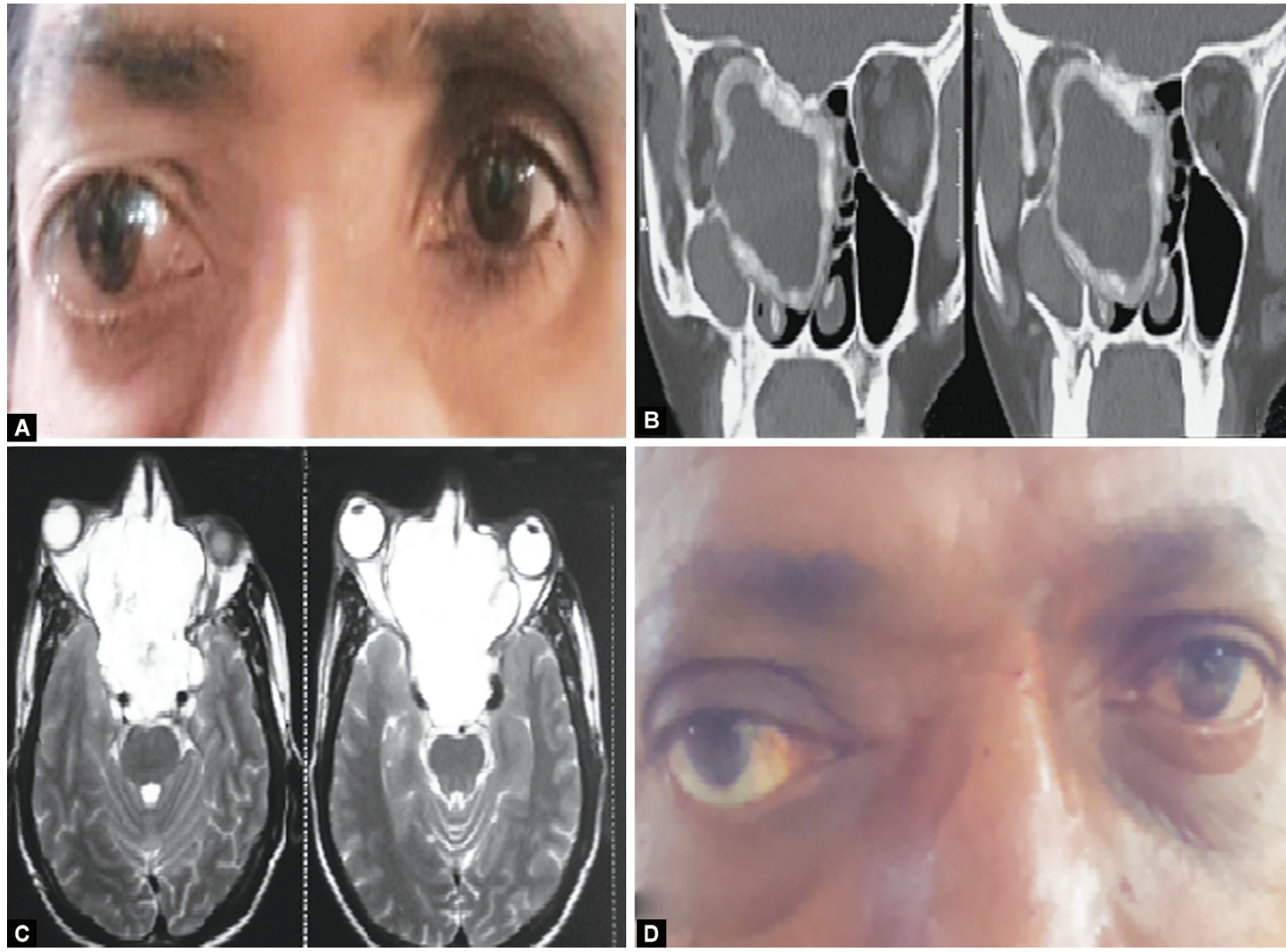

Figs 2A to D: (A) Clinical image of right eye proptosis - meningioma; (B) CT image of the extent of ethmoidal meningioma; (C) MRI image of the extent of myxoma of nasal septum; (D) Clinical image of right eye proptosis 

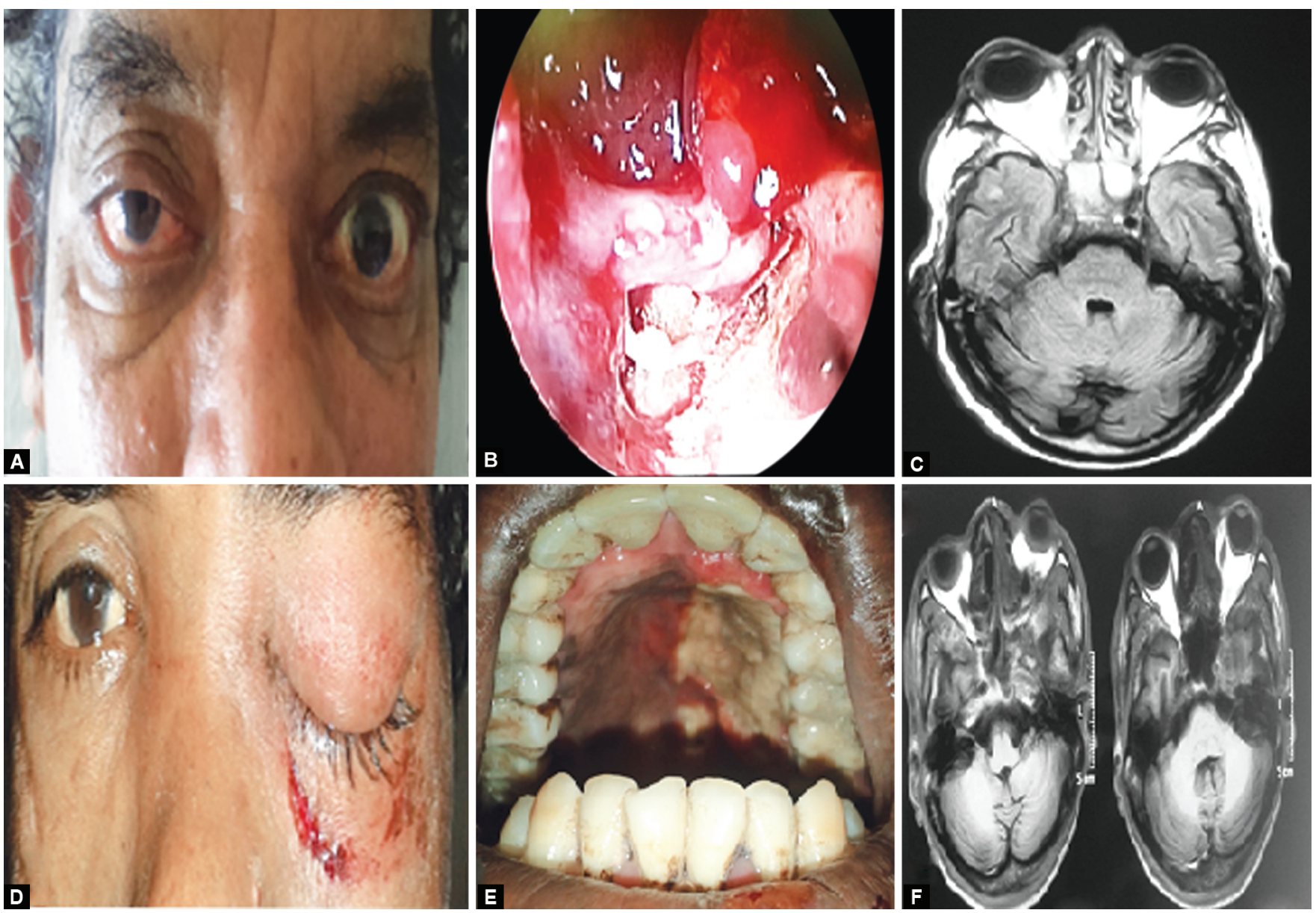

Figs 3A to F: Myxoma of nasal septum 2D clinical image of right eye proptosis

of the eye. Concomitantly, the patient had left-sided maxillary necrosis. Plain CT and MRI brain showed left frontal, maxillary, ethmoid, and sphenoid sinusitis with the extraconal extension of inflammation into left orbit causing proptosis of the left eyeball and orbital cellulitis along with thrombosis of the left carotid siphon and cavernosal internal carotid artery with multifocal acute infarcts in the left cerebral hemisphere. The pus culture and sensitivity found the offending agent to be Staphylococcus epidermidis sensitive to linezolid and was treated with endoscopic sinus surgery, debridement of the maxillary slough, and closure of maxillary sinus fistula with antibiotic cover. This was similar to a case report by Korean researchers Yeo et al. ${ }^{14}$ who also had a case of cavernous sinus thrombosis secondary to dental infection and was managed by intravenous antibiotics and pus drainage (Fig. 3).

Carotid-cavernous fistulas (CCFs) are anomalous communications between the carotid arterial system and the venous cavernous sinus. They can arise because of spontaneous or trauma causes. Traumatic CCFs may occur after either blunt or penetrating head trauma, ${ }^{15}$ as was the cause in our case. Their clinical presentation is related to their size and to the type of venous drainage, which can lead to various symptoms, such as visual loss, proptosis, bruit, etc. Our case had visible pulsatile proptosis and was treated with fistula closure. The case of rhabdomyosarcoma underwent total exenteration followed by chemoradiotherapy. The patient expired after 6 months and was the only mortality in our series.

\section{CONCLUSION}

The varied etiology of proptosis in these cases was intriguing and highlights the importance of knowledge of causes of proptosis in ENT. One of the major causes of unilateral proptosis is lesions (including malignancies) arising from the periorbital region. A good work up and with surgical management resulted in good recovery of the patients with only one mortality.

\section{Clinical Significance}

Thus, though proptosis is an orbital symptom a thorough and complete ENT examination is necessary for detecting such pathology caused by otorhinolaryngological causes, early. Managing them at an early stage improves the morbidity and mortality of the patients suffering from such lesions.

\section{ACKnOWLedgments}

We would like to thank the Department of Ophthalmology and Department of Pathology for all the help and support in providing the clinical material for the writing of this research article.

\section{References}

1. Keche $P$, Nitnaware $A Z$, Mair $M$, et al. A study of tumours giving rise to unilateral proptosis. Indian J Otolaryngol Head Neck Surg 2013;65(Suppl 1):6-13. DOI: 10.1007/s12070-011-0353-0. 
2. Rajenderkumar D. A study on etio-pathology of proptosis in otorhinolaryngology. Indian J Otolaryngol Head Neck Surg 1999;51(4):1-5. DOI: 10.1007/BF03022705.

3. Rosen FS, Ryan MW. (2004) Surgery for exophthalmos. Grand round presentation UTMB, Department of Otolaryngology. http:/1www. emedicine.com/ent.

4. Venugopal M, Sagesh M. Proptosis: rhe ENT surgeon's perspective. Indian Journal of Otolaryngology and Head \& Neck Surgery 2013;65(Suppl 2):247-250. DOI: 10.1007/s12070-011-0367-7.

5. Sinha V, Bhardwaj D, George A, et al. Proptosis through eyes of E.N.T. Surgeon. Indian J Otolaryngol Head Neck Surg 2005;57(3):207-209. DOI: 10.1007/BF03008015.

6. Kimmelman CP, Korovin GS. Management of paranasal sinus neoplasm invading the orbit. Otolaryngologic Clinics of North America 1988;21(1):77-92.

7. Calcaterra TC, Hepler RS, Hanafee WN. Unilateral exophthalmos. Laryngoscope 1974;2(2):231-242. DOI: 10.1288/00005537-19740200000004.

8. Wright. Annals of royal college of surgeons. England 1970;47:323.
9. Frazell $\mathrm{BL}$, Lewis JS. Cancer of nose and paranasal sinusesCancer 1963;16:1293-1313.

10. Zaidi SH. Unilateral proptosis in ENT practices. J Pak Med Assoc (JPMA) 1991;41(10):248-250.

11. Johnson LN, Krohel GB, Yeon EB. Sinus tumours invading the orbit. Opthalmology 1984;91(3):208-217. DOI: 10.1016/S01616420(84)34300-7.

12. Popovic D, Milisavljevic D. Malignant tumours of the maxillary sinus - a ten years experience. Med Biol 2004;11(1):31-34.

13. Bhansali $A$, Bhadada $S$, Sharma $A$, et al. Presentation and outcome of rhino-orbital-cerebral Mucormycosis in patients with diabetes. Postgrad Med J 2004;80(949):670-674. DOI: 10.1136/ pgmj.2003.016030.

14. Yeo G-S, Kim HY, Kwak E-J, et al. Cavernous sinus thrombosis caused by a dental infection: a case report. J Korean Assoc Oral Maxillofac Surg 2014;40(4):195-198. DOI: 10.5125/jkaoms.2014.40.4.195.

15. Karaman E, Isildak $\mathrm{H}$, Haciyev $\mathrm{Y}$, et al. Carotid-cavernous fistula after functional endoscopic sinus surgery. J Craniofac Surg 2009;20(2):556558. DOI: 10.1097/SCS.0b013e31819ba1e8. 